\title{
The genomes of two parasitic wasps that parasitize the diamondback moth
}

\author{
Min Shi ${ }^{1,2 \dagger}$, Zhizhi Wang ${ }^{1,2+}$, Xiqian $\mathrm{Ye}^{1,3+}$, Hongqing Xie ${ }^{4 \dagger}$, Fei $\mathrm{Li}^{1,2 \dagger}$, Xiaoxiao Hu${ }^{4,5+}$, Zehua Wang ${ }^{1,2}$, \\ Chuanlin Yin ${ }^{1,2}$, Yuenan Zhou ${ }^{1,2}$, Qijuan Gu, ${ }^{1,2}$, Jiani Zou ${ }^{1,2}$, Leqing Zhan ${ }^{1,2}$, Yuan Yao ${ }^{6}$, Jian Yang ${ }^{1,2}$, Shujun Wei \\ Rongmin $\mathrm{Hu}^{1,2}$, Dianhao Guo ${ }^{1,2}$, Jiangyan Zhu ${ }^{1,3}$, Yanping Wang ${ }^{1,3}$, Jianhua Huang ${ }^{1,2^{*}}$, Francesco Pennacchio ${ }^{8}$, \\ Michael R. Strand ${ }^{9}$ and Xuexin Chen ${ }^{1,2,3^{*}}$ (D)
}

\begin{abstract}
Background: Parasitic insects are well-known biological control agents for arthropod pests worldwide. They are capable of regulating their host's physiology, development and behaviour. However, many of the molecular mechanisms involved in host-parasitoid interaction remain unknown.

Results: We sequenced the genomes of two parasitic wasps (Cotesia vestalis, and Diadromus collaris) that parasitize the diamondback moth Plutella xylostella using Illumina and Pacbio sequencing platforms. Genome assembly using SOAPdenovo produced a $178 \mathrm{Mb}$ draft genome for C. vestalis and a $399 \mathrm{Mb}$ draft genome for D. collaris. A total set that contained 11,278 and 15,328 protein-coding genes for $C$. vestalis and D. collaris, respectively, were predicted using evidence (homology-based and transcriptome-based) and de novo prediction methodology. Phylogenetic analysis showed that the braconid C. vestalis and the ichneumonid D. collaris diverged approximately 124 million years ago. These two wasps exhibit gene gains and losses that in some cases reflect their shared life history as parasitic wasps and in other cases are unique to particular species. Gene families with functions in development, nutrient acquisition from hosts, and metabolism have expanded in each wasp species, while genes required for biosynthesis of some amino acids and steroids have been lost, since these nutrients can be directly obtained from the host. Both wasp species encode a relative higher number of neprilysins (NEPs) thus far reported in arthropod genomes while several genes encoding immune-related proteins and detoxification enzymes were lost in both wasp genomes.

Conclusions: We present the annotated genome sequence of two parasitic wasps C. vestalis and D. collaris, which parasitize a common host, the diamondback moth, P. xylostella. These data will provide a fundamental source for studying the mechanism of host control and will be used in parasitoid comparative genomics to study the origin and diversification of the parasitic lifestyle.
\end{abstract}

Keywords: Cotesia vestalis, Diadromus collaris, Parasitic wasps, Genome, Transcriptome

\section{Background}

Parasitic insects, particularly the parasitic wasps, are a large group of animals [1-4]. As adults, most species feed on nectar, while larvae feed as parasites on other arthropods. Adult females of parasitic wasps usually lay their eggs on or inside the body of a host, which usually

\footnotetext{
* Correspondence: jhhuang@zju.edu.cn; xxchen@zju.edu.cn

${ }^{\dagger}$ Min Shi, Zhizhi Wang, Xiqian Ye, Hongqing Xie, Fei Li and Xiaoxiao Hu contributed equally to this work.

${ }^{1}$ Institute of Insect Sciences, College of Agriculture and Biotechnology, Zhejiang University, Hangzhou 310058, China

Full list of author information is available at the end of the article
}

dies when offspring complete their development [2, 3]. Parasitic wasps are major natural enemies of a vast number of arthropod species in many orders [4]. Many species are also widely used as biological control agents of pests in agricultural and forest ecosystems [5, 6]. Most wasps have narrow host ranges, successfully develop in only one or a few species, and also parasitize only one life stage of their host (egg, larva, pupa, or adult) while many wasps share a common host species. Parasitic wasps that lay their eggs on hosts usually produce progeny that feed as ectoparasites, while species that lay

(c) The Author(s). 2019 Open Access This article is distributed under the terms of the Creative Commons Attribution 4.0 International License (http://creativecommons.org/licenses/by/4.0/), which permits unrestricted use, distribution, and reproduction in any medium, provided you give appropriate credit to the original author(s) and the source, provide a link to the Creative Commons license, and indicate if changes were made. The Creative Commons Public Domain Dedication waiver (http://creativecommons.org/publicdomain/zero/1.0/) applies to the data made available in this article, unless otherwise stated. 
their eggs in hosts produce progeny that feed as endoparasites [7]. Parasitic wasps are also either solitary, producing a single offspring per host, or gregarious and produce multiple offspring per host [7]. Parasitic wasps usually produce a number of virulence factors following oviposition that benefit offspring by altering the growth, development and immune defenses of hosts. The sources of these virulence factors include venom [8], symbiotic polydnaviruses (PDVs) [9-12], and teratocytes [13].

The genomes of more than 15 parasitic wasp species that parasitize different hosts have been sequenced (www. ncbi.nlm.nih.gov). These include Nasonia vitripennis (Hymenoptera: Pteromalidae), which is an ectoparasitoid that parasitizes the pupal stage of selected Diptera [14], Microplitis demolitor (Hymenoptera: Braconidae), which is an endoparasitoid that parasitizes the larval stage of selected species of Lepidoptera [15], and Fopius arisanus, which is an endoparasitoid that parasitizes larval stage Diptera in the family Tephritidae [16]. Collectively, these data provide several insights into parasitoid wasp biology. In contrast, no studies have examined the genomes of different species that parasitize the same host. Here, we sequenced two endoparasitoids in the superfamily Ichneumonoidea that parasitize the diamondback moth, Plutella xylostella L. (Lepidoptera: Yponomeutidae), which is a major worldwide pest of cruciferous crops (Fig. 1) [17, 18]. Cotesia vestalis (Haliday) is a solitary, larval endoparasitoid in the family Braconidae (Braconidae: Microgastrinae) that produces venom, a PDV named $C$. vestalis bracovirus (CvBV) and teratocytes. Larvae of $P$. xylostella parasitized by $C$. vestalis exhibit greatly reduced weight gain, delayed larval development and disabled cellular and humoral immune defences [19-21]. Diadromus collaris (Gravenhorst), is in the family Ichneumonidae (Ichneumonidae: Ichneumoninae) and is a solitary pupal endoparasitoid. $D$. collaris produces only venom. $P$. xylostella pupae parasitized by $D$. collaris fail to develop into adults and exhibit suppressed humoral and cellular immune defences [21].

In this study, we present the annotated genome sequence of two parasitic wasps $C$. vestalis and D. collaris, which parasitize a common host, the diamondback moth, P. xylostella. The gathered genomic data and transcriptome datasets collected from varying developmental stage and tissues will significantly expand our comprehension of the evolutionary history of parasitic wasps and their interactions with the common host, P. xyostella.

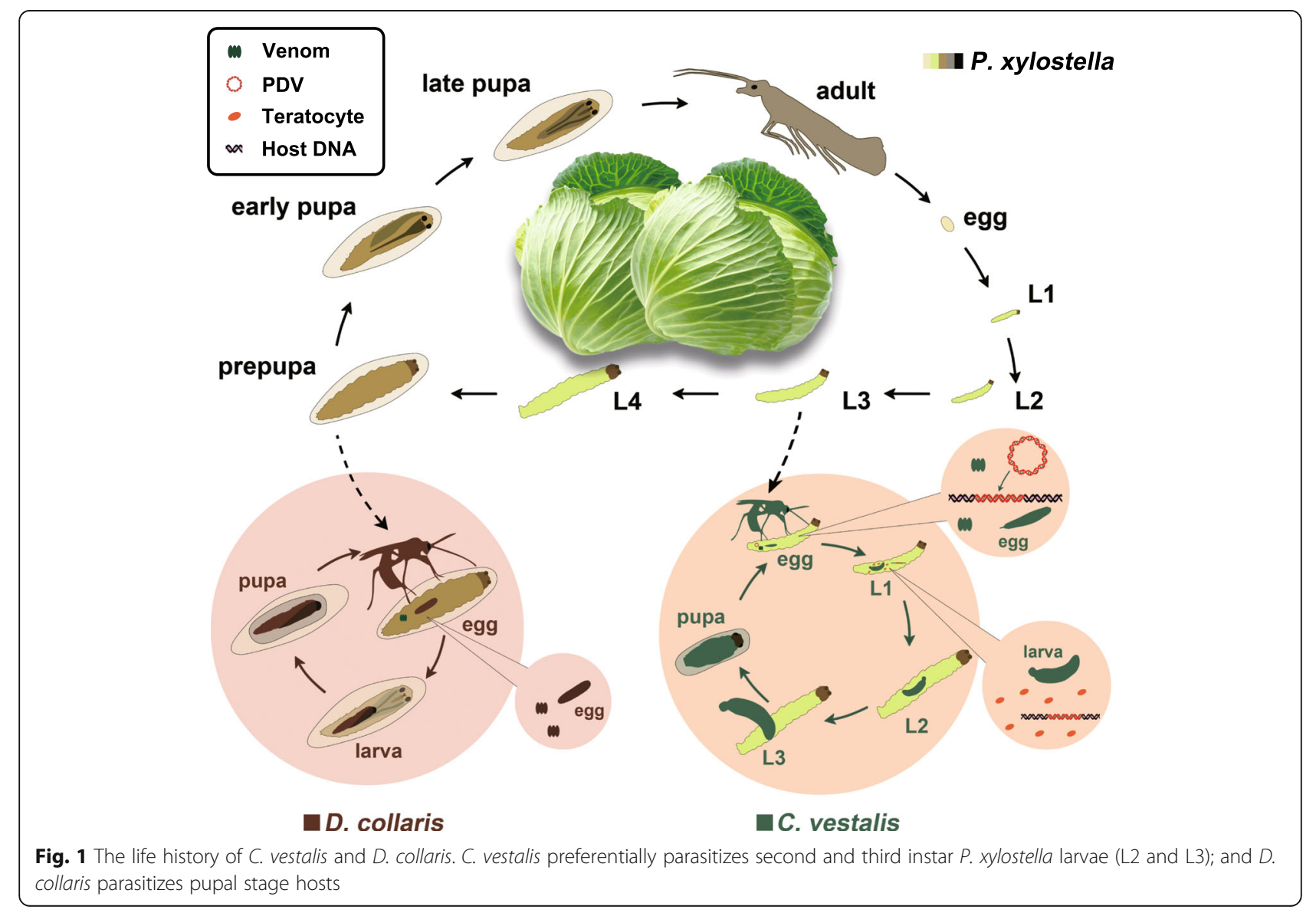




\section{Results}

\section{Genome assembly and gene information}

The whole genome sequencing was performed by combining Illumina Solexa sequencing based on HiSeq 2000 platform (Illumina, San Diego, CA, USA) and the LongRead Single Molecule Real-Time (SMRT) sequencing based on PacBio Sequel platform (Pacific Biosciences, Menlo Park, CA, USA) in consideration of cost and the low heterozygosity of wasp genome [22, 23]. In total, we obtained $36.10 \mathrm{~Gb}$ of raw data $(32.05 \mathrm{~Gb}$ from Illumina platform and $4.05 \mathrm{~Gb}$ from PacBio platform) for C. vestalis, and $65.91 \mathrm{~Gb}$ of raw data $(61.05 \mathrm{~Gb}$ from Illumina platform and $4.86 \mathrm{~Gb}$ from PacBio platform) for D. collaris (Additional file 1: Table S1). After filtering steps, $25.55 \mathrm{~Gb}(127.78 \times)$ from $C$. vestalis and $49.19 \mathrm{~Gb}$ (120.86x) from $D$. collaris were assembled using SOAPdenovo V2.04 [24] (Additional file 1: Table S2). These data were further assembled into a $178 \mathrm{Mb}$ draft genome for $C$. vestalis and a $399 \mathrm{Mb}$ draft genome for $D$. collaris, which were consistent with genome size estimates generated by $\mathrm{k}$-mer analysis (Table 1; Additional file 1: Figure S1). Genome assemblies for $C$. vestalis and $D$. collaris yielded scaffold $\mathrm{N} 50 \mathrm{~s}$ that were $2.60 \mathrm{Mb}$ and $1.03 \mathrm{Mb}$, respectively (Additional file 1: Table S3). We then checked the distribution of sequencing depth against GC content to infer the abundance of potential contamination of bacteria. As for GC content, compared with $C$. vestalis (29.96\%), D. collaris has a higher GC content, around 37\% (Table 1, Additional file 1: Figure $\mathrm{S} 2$ ). The bacterial contaminant reads in genome data of C. vestalis (Additional file 1: Figure S2) were filtered out after the assembling procedure. All transcripts were mapped to genome assemblies by BLAT with default parameters, resulting $91.7 \%$ transcripts of C. vestalis and $98.1 \%$ of $D$. collaris were found in the assembled genome, respectively (Additional file 1: Table S4). The

Table 1 Assembled Genomes and Gene Sets for C. vestalis and D. collaris

\begin{tabular}{lll}
\hline & C. vestalis & D. collaris \\
\hline Contig N50 (bp) & 51,333 & 25,941 \\
Scaffold N50 (Kb) & 2609.601 & 1030.36 \\
Quality control (covered by assembly) & \\
Genome size (Mb) & 178.55 & 399.17 \\
Number of scaffolds & 1437 & 2731 \\
BUSCO ( $n=1658)(\%)$ & $C^{a}: 96.7 \%, F: 2.4 \%$ & C: $99.2 \%, F: 0.3 \%$ \\
Genomic features & & \\
Repeat (\%) & 24 & 37 \\
G + C (\%) & 29.96 & 37.37 \\
Gene annotation & \\
Number of genes & 11,278 & 15,328 \\
\hline${ }^{a}$ C: complete BUSCOs; F: fragmented BUSCOs
\end{tabular}

quality of the assembly was further checked by Benchmarking Universal Single-Copy Orthologs BUSCO v3.0.2 [25] with insectdbV9 as referenced dataset. The recovered genes are classified as 'complete' when their lengths are within two standard deviations of the BUSCO group mean length. BUSCO analysis indicated the complete recovered genes for each species was greater than $96.7 \%$ (Table 1). These metrics strongly supported the overall quality of genome assemblies.

A total of 11,278 protein-coding genes for C. vestalis and 15,328 for $D$. collaris were identified by de novo and evidence-based (homology-based and transcriptomebased) prediction methods (Table 1, Additional file 1: Table S5 and S6). About 85\% of the inferred proteins for C. vestalis and $76.31 \%$ for D. collaris were annotated using the databases of KEGG, GO, TrEMBL, SWISSPROT and InterPro (Additional file 1: Table S7). Gene numbers were higher than for Apis mellifera $(10,660)$, but lower than for $N$. vitripennis $(17,084)$. As estimated by homology-based and de novo prediction methods, repetitive DNA accounted in D. collaris genome assembly (37\%) was higher than that in C. vestalis (24\%) (Additional file 1: Table S8), indicating the partial reason for the larger genome size of $D$. collaris. The total size of transposable elements (TEs) approached 31.1 $\mathrm{Mb}(17.4 \%$ of genome) for C. vestalis and $119.5 \mathrm{Mb}$ (29.93\% of genome) for D. collaris (Additional file 1: Table S8). TE diversity in D. collaris is $17 \%$ higher than that in $N$. vitripennis (66 Mb, 22\% of genome) and is 10 -fold higher than that in A. mellifera $(6.2 \mathrm{Mb}, 2.8 \%$ of genome). We sequenced small RNA of these two wasps by constructing small RNA libraries and used prediction software to identify a final set of 176 miRNAs in $C$. vestalis and 117 miRNAs in $D$. collaris. Both numbers are relatively higher than those in $N$. vitripennis (98 miRNAs) and A. mellifera (94 miRNAs), but much lower than those in D. melanogaster (165 miRNAs) (Fig. 2 and Additional file 2: Table S9). Beside 55 known miRNAs that conserved across these two wasp genomes, we also identified total 47 novel, previously uncharacterized miRNA, 14 of them were specific to $C$. vestalis and the rest were specific to $D$. collaris. The small number of conserved miRNAs and relatively large number of novel miRNAs was somewhat surprising in light of each species developing in the same host and living in the same habitats where $P$. xylostella occurs.

\section{Genome phylogeny and comparisons}

We compared orthologous gene pairs identified in $C$. vestalis and $D$. collaris to 8 other hymenopteran species, 8 other insect species in diverse orders, and 1 mite species (Tetranychus urticae) in the order Trombidiformes (Fig. 3a). Over $85 \%$ of the genes in each of the wasp species we sequenced were orthologous to genes in one or 


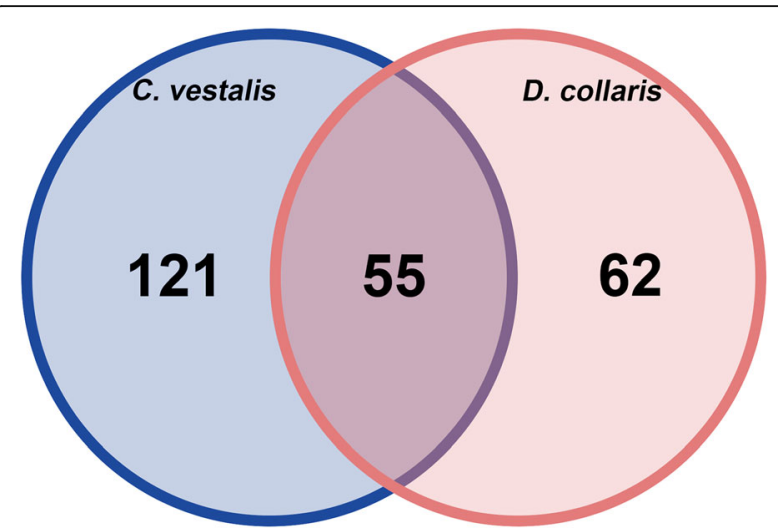

Fig. 2 Venn diagram of the distribution of unique and shared miRNAs across $C$. vestalis and D. collaris. A final set of 176 miRNAs in C. vestalis and 117 miRNAs in D. collaris. Strikingly, 55 miRNAs were conserved in these two wasp genomes

more other species. The number of single-copy genes in C. vestalis and D. collaris was 659 (4.9\%) and $665(3.8 \%)$, respectively. These two wasps contained more than 6000 many-to-many universal genes, which accounted for 37$47 \%$ of the total gene sets (Fig. 3a). The wasp gene sets showed a significantly higher proportion of many-tomany orthologues than single-copy genes, suggesting that duplication occurs more frequently in the universal orthologues than in insect-specific genes.

We constructed a phylogenetic tree with 262 universal single-copy orthologues using maximum likelihood methods. Consistent with prior analyses [26], our results supported that these two parasitoid wasps in the superfamily Ichneumonoidea diverged from the Aculeata (bees and ants) approximately 140 million years ago, and the braconid $C$. vestalis and the ichneumonid $D$. collaris diverged approximately 124 million years ago (Fig. 3a). In total, we identified 8311 gene families in $C$. vestalis and 9063 in D. collaris. The number of unique gene/ gene families in $C$. vestalis and D. collaris was 474 and 1060, respectively (Fig. 3b). C. vestalis shared 258 gene families with $D$. collaris (Fig. $3 b$ ). The occurrence of the same gene families in different parasitoid species could be a consequence of a common adaptive pathway to parasitic lifestyle. C. vestalis, D. collaris and A. mellifera encoded a similar proportion (about 60\%) of single-copy orthologous that shared amino acid identities (Fig. 3c). Based on ratio of syntenying genes, very high degrees of microsynteny were observed between $C$. vestalis and $D$. collaris orthologs (Fig. 3d, Additional file 1: Table S10 and S11), much more than that between $C$. vestalis and $A$. mellifera, D. collaris and A. mellifera (Additional file 1: Table S10 and S11), which indicated numerous chromosomal rearrangements have occurred in hymenopteran species since diverged from their last shared ancestor. The synteny shared between $C$. vestalis and $D$. collaris reflect more conserved genes maintained across these two species.

\section{Gene family expansions and gene losses}

We used CAFÉ [27] to examine gene family expansions and contractions in this study. When compared to other arthropods, 30 gene families in C. vestalis and 65 in D. collaris exhibited significant expansions, while 23 gene families in $C$. vestalis and 3 in D. collaris were contracted $(P<0.05)$ (Additional file 3: Table S12). During further analysis of selected expanding gene families $(P=0)$ for analysis (Fig. 4), we found neprilysins (NEPs) was expanded in these two species and also other two hymenopteran species, Diadegma semiclausum and M. demolitor (Figs. 4 and 5a). We investigated the expression pattern of NEP genes from $C$. vestalis at different developmental stages via RNA-seqbased differential expression analysis (Fig. 5b). Among the 28 NEP genes in C. vestalis, more than half were highly expressed in eggs and larvae. Several gene families, such as CDK1, SKP1, PLA2, RNASET2 and CA7, associated with developmental regulation showed expansions in $C$. vestalis. In $D$. collaris, we observed the expansion of histone genes and other genes encoding enzymes with functions in trehalose transport (TRET) and fatty acid metabolism, such as fatty acid synthase $(F A S)$, stearoyl-CoA desaturase $(S C D)$, and elongation of very long chain fatty acids protein (ELOVL) (Fig. 4).

We also observed that certain contracted gene families in $C$. vestalis were expanded in D. collaris, such as carboxylesterase, $S C D$, histone and ribonucleoside-diphosphate reductase beta chain. The expansion and contraction of the same gene family maybe, to some extent, reflect the different lifestyle of these two wasps. Wasps are carnivorous animals that evolved from a branch of herbivorous insects. It is reasonable that these two wasps lacked a number of enzymes required to synthesize nine essential amino acids (glutamate, histidine, isoleucine, leucine, lysine, methionine, phenylalanine, tryptophan, and valine), and two non-essential amino acids (arginine and tyrosine) (Additional file 1: Figure S3).

\section{Gene families associated with immunity}

The $C$. vestalis and D. collaris genomes contained all components of the major insect immune pathways (Additional file 1: Table S13). However, comparisons to the other Hymenoptera (Nasonia vitripennis, Apis mellifera), Diptera (Drosophila melanogaster, Anopheles gambiae), and the host P. xylostella, we noticed the variation in the composition of certain immune gene families. For example, $C$. vestalis, $D$. collaris, and A. mellifera encoded smaller numbers of pattern recognition genes (peptidoglycan-recognition proteins (PGRPs), gram-negative bacteria binding proteins (GNBPs), galectins, fibrinogen-related proteins (FREPs) and C-type lectins than $N$. vitripennis, $D$. 


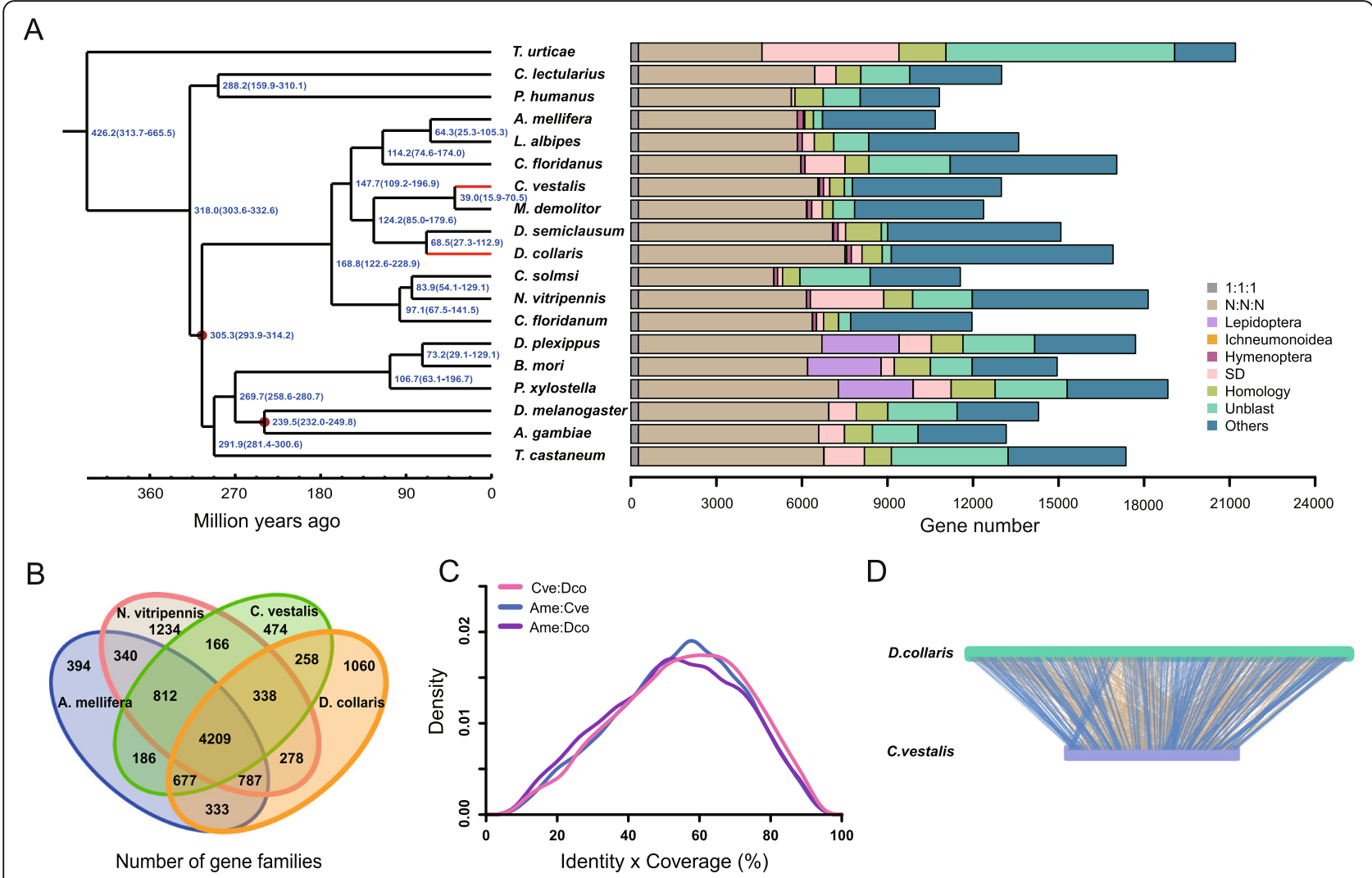

Fig. 3 Phylogenetic tree and orthologue assignments of 19 arthropod genomes. a The phylogenetic tree was constructed from 262 single-copy genes using maximum likelihood methods. Red points on the internal nodes indicate fossil calibration times in the analysis. Blue numbers indicate estimated divergence times (Mya, million years ago). The red branches identify the two wasps sequenced in this study. The different types of orthologous relationships are shown. "1:1:1" = universal single-copy genes, although the absence in a single genome is tolerated; "N:N:N" = orthologues in all genomes, although the absence in less than 2 genomes is tolerated. "Ichneumonoidea" = ichneumonoid-specific genes, although the absence in a single genome is tolerated; "Lepidoptera" = lepidopteran-specific genes, although the absence in a single genome is tolerated; "SD" = species-specific duplicated genes; "Homology" = genes with an e-value less than 1e-7 as determined by BLAST, although they do not cluster into a gene family; "Unblast" = species-specific genes that are not observed in other species with e-values less than 1e-7 as determined by BLAST; and "Others" = orthologs that do not fit into the other categories. b Shared and unique gene families in C. vestalis, D. collaris, N. vitripennis and A. mellifera are shown in the Venn diagram. c Comparison of the distributions for identity values of orthologous genes in C. vestalis, D. collaris and A. mellifera. $\mathbf{d}$ Microsynteny in C. vestalis and D. collaris determined by tracking the gene positions. In addition to C. vestalis and D. collaris, species names and ordinal affiliations for the arthropods in the data set are: Anopheles gambiae (Diptera), Apis mellifera (Hymenoptera), Bombyx mori (Lepidoptera), Copidosoma floridanum (Hymenoptera), Cimex lectularius (Hemiptera), Camponotus floridanus (Hymenoptera), Ceratosolen solmsi (Hymenoptera), Danaus plexippus (Lepidoptera), D. melanogaster (Diptera), Diadegma semiclausum (Hymenoptera), Lasioglossum albipes (Hymenoptera), Microplitis demolitor (Hymenoptera), Plutella xylostella (Lepidoptera), Nasonia vitripennis (Hymenoptera), Pediculus humanus (Phthiraptera), Tribolium castaneum (Coleoptera), and Tetranychs urticae (Trombidiformes)

melanogaster, A. gambiae, and P. xylostella. The overall lower number of antimicrobial peptide genes (AMPs) were also found in C. vestalis, D. collaris, and A. mellifera larvae. However, these trends are not fully uniform given the greater number of defensin genes in C. vestalis (11) and $D$. collaris (7) relative to $N$. vitiripennis (5). In addition, 27 putative inhibitors of apoptosis (IAP) genes were identified in $D$. collaris, while other species contained only 3 to 7 (Additional file 1: Table S13).

Transcriptome analyses in $C$. vestalis showed the changes in the expression profiles of many immune-related genes during development (Additional file 4: Table S14); in particular, the expression levels of immune genes such as defensin, serpin and C-type lectins were significantly abundant in larvae and teratocytes of $C$. vestalis. We also determined that 8 of 27 iap genes in $D$. collaris were expressed in venom glands.

\section{Gene families associated with xenobiotic detoxification} $C$. vestalis and $D$. collaris together with other hymenopterans (C. solmsi, N. vitripinnis and A. mellifera) encoded less glutathione-S-transferases (GSTs) when compared with other arthropods (Additional file 1: Table S13). C. vestalis and $A$. mellifera also encoded less cytochrome P450s (CYPs) (Additional file 1: Table S15). In contrast, $D$. collaris encoded a very close number of P450s and 


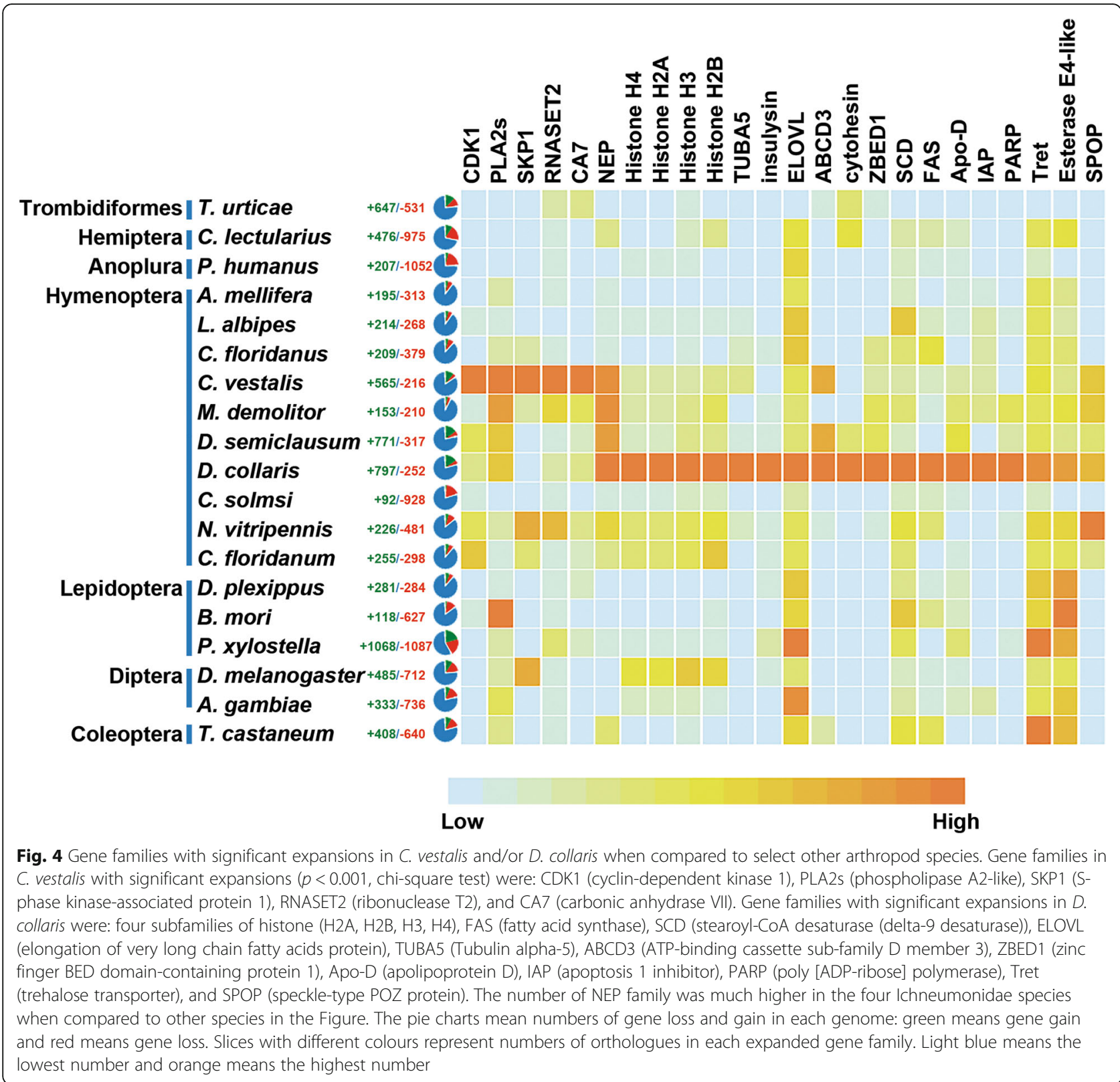

carboxylesterases as $N$. vitripinnis, which were together broadly comparable to several other arthropods (Additional file 1: Table S13). Transcriptome analyses revealed that most of these detoxification enzyme genes were expressed in different life stages of $C$. vestalis (Additional file 4: Table S14). While no transcriptome data could be generated for different life stages of $D$. collaris, we speculate the detoxification enzyme genes identified in this species are likely expressed in similar stage-specific patterns to other hymenopterans.

\section{Discussion}

More than 100 insect genomes have been sequenced during the last two decades [28], which provided valuable information to expand our understanding for the biodiversity of insect habits, behaviors and long-term evolutionary relationship. Yet, there are still many species with important roles in agriculture, which certainly are worth made thorough research. In this study, we report two phylogenetically related wasp genomes, $C$. vestalis and D. collaris, which are responsible for regulating the populations of a worldwide pest, $P$. xylostella. In spite of their strong genetic divergence, a small number of common features indicate that the genome of the wasps is, in certain ways, shaped by endoparasitism. This knowledge can be useful in revealing genomic convergence of parasitic wasps associated with the same host and the convergence to endoparasitic lifestyle. 


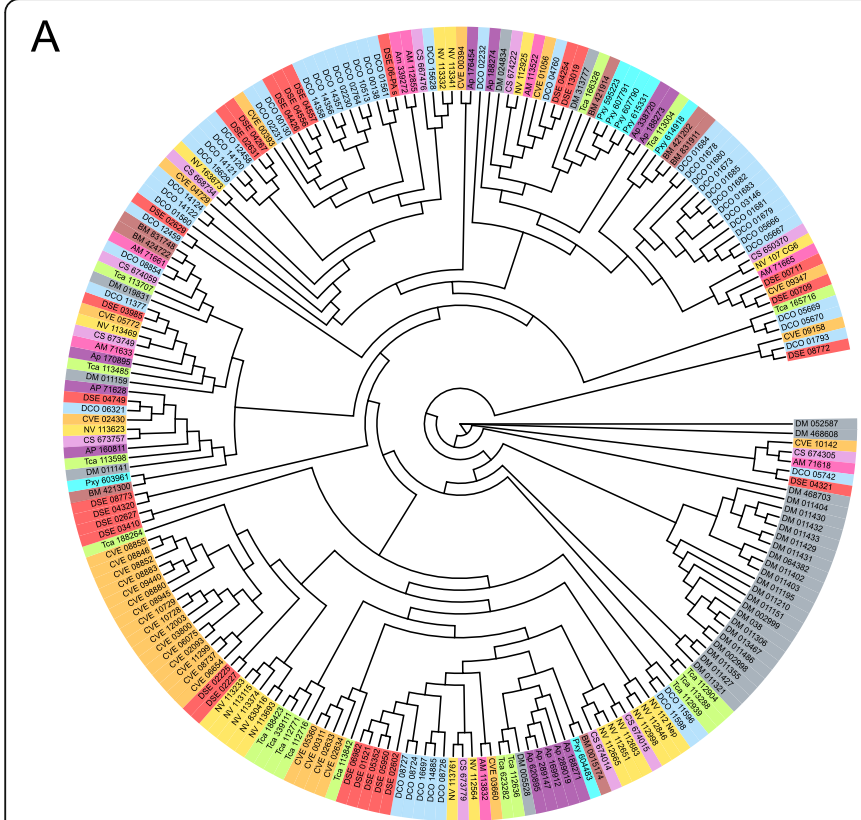

\section{B}

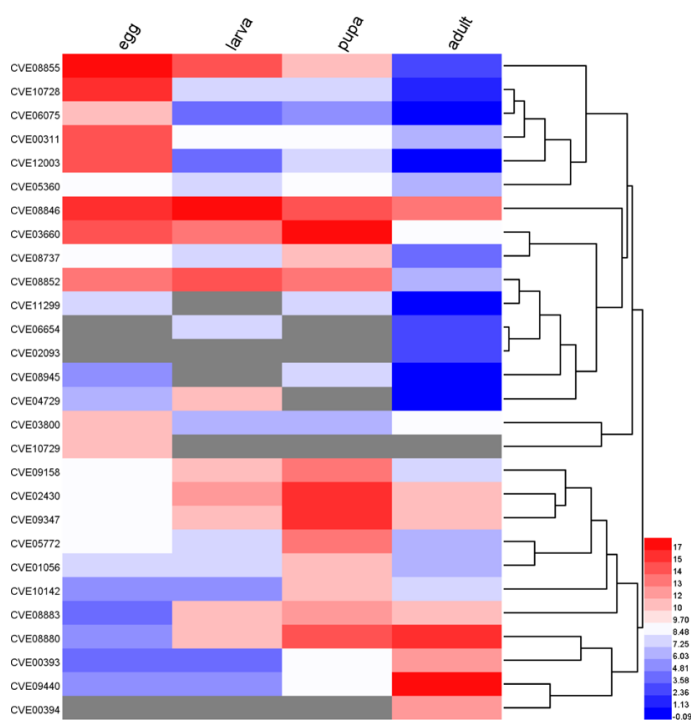

Fig. 5 The phylogenetic relationships of neprilysins in insects and their expression levels in different stage of $C$. vestalis. a The phylogenetic relationships of neprilysins in insects. 216 neprilysins of 11 species (A. mellifera, A. pisum, B. mori, C. solmsi, C. vestalis, D. collaris, D. melanogaster, D. semiclausum, N. vitripennis, P. xylostella, T. castaneum) were used to construct the phylogenetic tree by maximum likelihood method. $\mathbf{b}$ Heatmap showing expression levels of all the NEP genes in different stage of $C$. vestalis. A total of 28 genes and two pseudugenes were found in $C$. vestalis genomes. Among the 28 NEP family genes in C. vestalis, more than half of them were highly expressed in parasitic periods (egg and larvae stages)

The whole genome sequencing generated the draft genome of C. vestalis $(178 \mathrm{Mb})$ and D. collaris $(399 \mathrm{Mb})$. A total set that contained 11,278 and 15,328 protein-coding genes for $C$. vestalis and $D$. collaris, respectively, was predicted using evidence and de novo prediction methodology. Over $85 \%$ of the genes in C. vestalis and D. collaris were orthologous to genes in one or more other species. The number of unique gene/gene families in $C$. vestalis and D. collaris was 474 and 1060, respectively, and C. vestalis shared 258 gene families with $D$. collaris. Based on ratio of syntenying genes, very high degrees of microsynteny were observed between $C$. vestalis and $D$. collaris orthologs. When compared to other arthropods, 30 gene families in C. vestalis and 65 in D. collaris exhibited significant expansions, while 23 gene families in $C$. vestalis and 3 in D. collaris were contracted.

The gene gains and losses of C. vestalis and D. collaris, in some cases, reflected their shared life history as endoparasites and in other cases are unique to particular species. Certain gene families with predicted functions in development, nutrient acquisition from hosts, and metabolism have expanded, while genes required for biosynthesis of some amino acids and steroids have been lost as a potential consequence of these resources being available from their shared host, P. xylostella. Both species encode the highest number of NEPs thus far reported in arthropod genomes. NEPs are metalloproteases in the M13 peptidase family, which in vertebrates degrade several peptide hormones [29, 30] and amyloid beta [31, 32]. Recent studies also implicate NEPs in inhibiting coagulation of vertebrate blood through inactivation of fibrinogen and suppressing melanisation, a response regulated by the phenoloxidase (PO) cascade [33, 34]. Interesting, NEP is a major component of the parasitoid Venturia canescens virus like particles inducing protection of parasitoid eggs against encapsulation [35, 36]. Given its diversity function in immunity, we speculate that NEP expansion in C. vestalis and $D$. collaris could probably reflect a conserved role in the Ichneumonidae but not exclude the possibility that the expansion of this gene family is involved in evasion of host immune defenses. The expanding gene families in $C$. vestalis, CDK1 and SKP1 may involve in cell cycle progression, signal transduction and transcription, while PLA2, RNASET2 and CA7 are enzymes that catalyze a number of different biochemical reactions [37, 38]. However, the biological significance of expanding gene families associated with developmental regulation in $C$. vestalis is unclear, because they could contribute to either wasp physiology or the altered physiology of hosts. In D. collaris, the expansion of histone genes potentially reflects the rapid development of this species [39] and the need to quickly produce sufficient amount of protein to coat the genome when replicated during the $\mathrm{S}$ phase of every cell cycle, and it is also consistent with the increase in rRNA 
copy number we found. Meanwhile, the expansion of encoding enzymes with functions in trehalose transport (TRET) and fatty acid metabolism properly suggests their roles in exploring and using of host nutrients by $D$. collaris [40].

The $C$. vestalis and $D$. collaris genomes contained all components of the major insect immune pathways, but the gene numbers are varied in the composition of certain immune gene families. The overall lower number of immune related genes probably reflects the reduced risks of pathogen exposure in the environments where larval-stage endoparasitoids (hosts) and A. mellifera (colonies) reside [41] relative to the microbial-rich environments where ectoparasitic $N$. vitripennis larvae (carrion), D. melanogaster larvae (decaying fruit), and P. xylostella larvae (cruciferous plants) develop. The significantly abundant expression profiles of many immunerelated genes, such as defensin, serpin and C-type lectins in larvae and teratocytes of $C$. vestalis revealed by transcriptome analyses indicated the need to protect immunosuppressed hosts from secondary bacterial infections [42]. Same abundant expression profiles of given iap genes in $D$. collaris venom glands indicated these IAPs could be involved in the functions of venom, maybe inhibit the apoptosis of venom gland cell to continuously produce venom proteins in a longer time, or they could be delivered into host cells to interfere pupal development of hosts [43, 44].

Xenobiotic detoxification involves the conversion of lipid-soluble substances to water-soluble, extractable metabolites [45]. The process of xenobiotic detoxification is primarily affected by CYPs, carboxylesterases and GSTs in insect, which are known to vary between species as a function of taxon and life history [46]. Parasitoids of herbivores have also been suggested to exhibit relatively poor capabilities in metabolizing xenobiotics [47]. The overall greater number of detoxification genes in $D$. collaris relative to $C$. vestalis also suggests a higher capacity to detoxify xenobiotics. This could reflect a lower capacity by $P$. xylostella pupae to detoxify xenobiotics than larvae, which has been selected for greater investment in xenobiotics metabolism in $D$. collaris. It is also possible the larger number of P450s and carboxylesterases encoded by $D$. collaris have functions outside of xenobiotic detoxification given the roles of these enzymes in other physiological processes. Differences in the total inventory of gene families likely reflect a combination of ancestry, since each wasp species resides in different families or subfamilies of the Ichneumonoidea, and life history associated with each species exhibiting differences in the life stage of $P$. xylostella they preferentially parasitize.

\section{Conclusion}

We presented the annotated genomes of the two endoparasitoid wasps $C$. vestalis and $D$. collaris that parasitized the same host $P$. xylostella using Illumina and Pacbio sequencing platforms. These data will be a fundamental resource for developing new methods of biological control for the diamondback moth and provide more insights into the evolutionary interactions between parasitic wasps and their host, i.e., the sequencing of the $C$. vestalis genomes provide the references needed for identifying $C$. vestalisproduced miRNAs and assessing their roles in parasitism of $P$. xylostella [48]. These genomes could also be used in comparative genomics analysis with the recently released genomes of other hymenopterans including closely related parasitic wasp species to study the origin and diversification of the parasitic lifestyle.

\section{Methods}

\section{Insect rearing}

Laboratory cultures of $C$. vestalis and D. collaris were maintained by parasitizing a laboratory culture of $P$. xylostella. Each wasp species was originally collected from the cabbage field $(30.3009 \mathrm{~N} 120.0870 \mathrm{E})$ in Hangzhou, China. The culture of $P$. xylostella was also established from field-collected material, and was subsequently maintained by feeding larvae on cabbage grown at $25^{\circ} \mathrm{C}, 65 \%$ relative humidity and a $14 \mathrm{~h}$ light: $10 \mathrm{~h}$ dark photoperiod. Adult wasps were fed a $20 \%(\mathrm{w} / \mathrm{v})$ honey solution. Each wasp species had been reared continuously for 5 years, spanning more than 100 generations, as inbred lines before the onset of this study.

\section{Genome sequencing}

A whole genome shotgun strategy was used to sequence the genomes of $C$. vestalis and D. collaris using the Illumina HiSeq 2000 platform (Illumina, San Diego, CA, USA). Long-Read Single Molecule Real-Time (SMRT) sequencing was also used for sequencing $C$. vestalis and D. collaris genomes performed on PacBio Sequel platform (Pacific Biosciences, Menlo Park, CA, USA) with P6 polymerase binding and $\mathrm{C} 4$ chemistry kits. DNA from a single wasp was insufficient for all sequencing runs. We thus extracted DNA from 2000 C. vestalis pupae, and 1000 adults from $D$. collaris using Qiagen DNA extraction kit. However, the use of inbred laboratory cultures ensured low levels of intraspecific sequence variation. To avoid sequence context bias, we constructed libraries with different insert sizes for HiSeq sequencing, including 2 paired-end sequencing libraries (170 bp, $500 \mathrm{bp})$ and 4 mate-pair libraries $(2 \mathrm{kbp}, 5 \mathrm{kbp}$, $10 \mathrm{kbp}$, and $20 \mathrm{kbp}$ ) for $C$. vestalis, and 3 paired-end sequencing libraries $(170 \mathrm{bp}, 500 \mathrm{bp}, 800 \mathrm{bp})$ and 4 mate-pair libraries (2 kbp, $5 \mathrm{kbp}, 10 \mathrm{kbp}$, and $20 \mathrm{kbp}$ ) for $D$. collaris. While for PacBio sequencing, we constructed library of $20 \mathrm{kbp}$ using the standard protocol. In total, we obtained $36.10 \mathrm{~Gb}$ of raw data for C. vestalis, and $65.91 \mathrm{~Gb}$ of raw data for $D$. collaris. 


\section{Transcriptome sequencing}

Samples were collected from four developmental stages (eggs, 2nd instar larvae, pupae, and adult females), teratocytes and venom glands of $C$. vestalis and venom glands of $D$. collaris for transcriptome sequencing. To avoid sample contamination by host material, wasp eggs and larvae were dissected from hosts and washed extensively in Petri dishes using TNH-FH medium (HyClone, Logan, UT, USA) before total RNA extraction. Teratocytes and venom glands were collected as previously described [21, 42]. Total RNA was isolated from the whole body using TRIzol reagent (Ambion, Foster City, CA, USA). RNA sequencing libraries were constructed using the Illumina mRNA-Seq Prep Kit (Illumina, San Diego, CA, USA). Oligo (dT) magnetic beads were used to purify mRNA molecules with poly (A). The mRNA was fragmented and was used as template, and random hexamers were used as anchor primers in the first-strand cDNA synthesis. The second-strand DNA was synthesized with DNA polymerase I to create double-stranded cDNA fragments. Double stranded cDNA was end repaired using T4 DNA polymerases and A-tailed using Klenow fragment lacking exonuclease activity. Illumina sequencing adapters were then added to the ends of double-stranded cDNAs and size selected by gel electrophoresis. Purified DNAs were then amplified by PCR followed by generation of $200 \mathrm{bp}$ paired-end libraries that were sequenced using Illumina HiSeq 2000 platform.

\section{Small RNA libraries sequencing}

We isolated 20 2nd instar larvae and of $C$. vestalis, teratocytes from 200 parasitized P. xylostella larvae and 20 3rd instar larvae of $D$. collaris from the parasitized $P$. xylostella pupae followed by washing each sample in TNM-FH medium three times. Total RNA was isolated using TRIzol reagent (Ambion, Foster City, CA, USA) followed by enrichment of 18-30 nt small RNAs using the PAGE method [49]. Small RNA sequencing libraries were constructed using TruSeq Small RNA Library Preparation Kits (Illumina, San Diego, CA, USA). Library sequencing was performed by Illumina HiSeq 2000. MicroRNA genes were inferred by miRdeep 2 software against the Rfam database of release 11.0 [50].

\section{Estimation of genome size}

$\mathrm{K}$-mer refers to an artificial sequence division of $\mathrm{K} \mathrm{nu-}$ cleotides iteratively from sequencing reads. A raw sequence read with $\mathrm{L}$ bp contains $(\mathrm{L}-\mathrm{K}+1) \mathrm{k}$-mers if the length of each $\mathrm{k}$-mer is $\mathrm{K} \mathrm{bp}$. The frequency of each $\mathrm{k}$ mer can be calculated from the genome sequence reads. K-mer frequencies along the sequence depth gradient follow a Poisson distribution in a given dataset. The genome size, G, is defined as G=K_num/K_depth, where the K_num is the total number of $\mathrm{k}$-mers and $\mathrm{K}$-depth is the frequency occurring more frequently than others. Short insert size libraries (170 bp and $500 \mathrm{bp}$ for C. vestalis and $500 \mathrm{bp}$ for $D$. collaris) were used to estimate the genome sizes with k-mer set as 17 .

\section{Genome assemblies}

To ensure the reliability of genome assembly, several types of reads were filtered. The filtering criteria was as following: (1) Reads from short insert-size libraries having an ' $\mathrm{N}$ ' over $2 \%$ of its length and the reads from large insert-size libraries having an 'N' over $5 \%$ of its length of C. vestalis. (2) Reads from both insert-size libraries having an 'N' over $2 \%$ of its length of $D$. collaris. (3) Reads from short insert-size libraries having more than $30 \%$ bases with quality $\leq 7$ and reads from large insert-size libraries having more than $40 \%$ bases with quality $\leq 7$ of C. vestalis. Reads from short insert-size libraries having more than $40 \%$ bases with quality $\leq 7$ and reads from large insert-size libraries having more than $60 \%$ bases with quality $\leq 7$ of $D$. collaris. (4) Reads with more than $10 \mathrm{bp}$ from the adapter sequence (allowing no more than two mismatches). (5) Small insert size paired-end reads that overlapped $\geq 10 \mathrm{bp}$ between the two ends. (6) Two paired-end reads that were identical (and thus considered to be the products of PCR duplication). (7) Reads having k-mer frequency $<4$ after correction (to minimize the influence of sequencing errors) of $D$. collaris.

The long read SMRT sequencing data was corrected using CANU (http://canu.readthedocs.org/) with default parameters [51]. After that, the corrected reads were used to extend the scaffolds assembled by SOAPdenovo V2.04, and the result was polished using Quiver (http:// www.pacbiodevnet.com/Quiver/).

After these filtering steps $25.55 \mathrm{~Gb}(127.78 \times)$ from $C$. vestalis and $49.19 \mathrm{~Gb}(120.86 \times)$ from $D$. collaris were assembled using to SOAPdenovo V2.04 [24]. Reads from short insert size libraries (ranging from 170 to $800 \mathrm{bp}$ ) were split into different optimized sizes of k-mers to construct de Bruijn graphs and then merged into contigs by k-1 non-mismatching overlap for two k-mers. We tested different $\mathrm{k}$-mer for each species to define the optimal K-mer for assembly. Based on the N50 and N90, the best assembly was achieved when $\mathrm{K}$ was set as 29-mer for $C$. vestalis and D. collaris. The paired-end information was subsequently used to link contigs into scaffolds from short insert sizes to long insert sizes. Gap close software KGF V1.19 and GapCloser V1.10 were used to fill gaps of $C$. vestalis and D. collaris. To assess assembly quality, high quality reads satisfying filtering criteria were aligned using SOAP2 with less than 3 mismatches. This yielded $95.9 \%(89.2 \times$ data $)$ and $91.8 \%(63.7 \times$ data $)$ coverage for the $C$. vestalis and $D$. collaris assemblies, respectively. Reads from the transcriptome data sets were assembled by SOAPdenovo 2 with a k-mer size of 
25. The quality of the assembly was checked using Benchmarking Universal Single-Copy Orthologs BUSCO v3.0.2 [25], using insectdbV9 as lineage dataset and reference.

\section{Repeat annotations}

Tandem Repeats Finder (TRF) was used to search tandem repeats in each assembled genome [52]. Transposable elements (TEs) were predicted in the assemblies by homology searching against RepBase, using RepeatProteinMask and RepeatMasker [53] with default parameters.

\section{Gene prediction and functional annotation}

We predicted gene sets in the assembled genomes using evidence (homology-based and transcriptome-based) and de novo prediction methodology. For homology-based prediction, A. mellifera, D. melanogaster, $N$. vitripennis, and Tribolium castaneum proteins were mapped onto the $C$. vestalis and $D$. collaris genomes using tblastn software [54]. Then, gene models were identified by mapping the homologous protein sequences against the tblastn hits detected in these two genomes using Genewise [55]. RNASeq data were mapped to the genome using TopHat [56], and transcriptome-based gene structures were obtained by cufflinks [56] (http://cufflinks.cbcb.umd.edu/). For de novo prediction, Augustus [57] and Genscan [58] were used to predict coding genes using appropriate parameters. GLEAN (http://sourceforge.net/projects/glean-gene/) was used to merge the gene sets, removing all genes with sequences less than 50 amino acids as well as those that only had de novo support. Finally, homology-based, de novo derived, and transcript gene sets were merged to form a comprehensive and non-redundant reference gene set. Gene functions were assigned based on the best match derived from each annotation by Blastp against the SwissProt and TrEMBL [59] databases. We annotated motifs and domains using InterPro [60] by searching against publicly available databases, including Pfam, PRINTS, PROSITE, ProDom, and SMART. Gene Ontology [61] information was retrieved from InterPro. We also mapped the reference genes to KEGG [62] pathway maps by searching KEGG databases and found the best hit for each gene.

\section{Identification of orthologues and synteny}

Similarities and differences among $C$. vestalis and $D$. collaris genes were assessed by best reciprocal hit of protein sequences using BLASTP with e-values $<0.01$ between any two pairs of species defined as orthologous counterparts. The similarity of genes was indicated as a density plot of aligned ratio and identity derived directly from Blastp. The amino acid identity of single-copy orthologous between $C$. vestalis and D. collaris was about 60\% (Fig. 3a). Syntenic blocks between C. vestalis and $D$. collaris were identified based on the orthologous gene order as described above. Specifically, syntenic blocks were defined as at least 3 orthologous counterparts that are both clustered (not interrupted by more than 5 genes) and located in continuous loci in a single scaffold for each species in a given pair of species.

\section{Phylogenetic tree and divergence time}

Coding sequences and protein data of C. vestalis, D. collaris, 8 other hymenopteran species including the publicly available genomes from four species of parasitic Hymenoptera ( $N$. vitripennis (family Pteromalidae), Ceratosolen solmsi (Agaonidae), M. demolitor (family Braconidae), and Camponotus floridanus (family Encyrtidae) and one genome for Diadegma semiclausum (Hellen) (family Ichneumonidae), which is also a larval-pupal parasitoid of the diamondback moth $P$. xylostella, generated by Illumina technology in our laboratory, 8 other insect species in diverse orders (Anopheles gambiae (Diptera), Bombyx mori (Lepidoptera), Cimex lectularius (Hemiptera), Danaus plexippus (Lepidoptera), D. melanogaster (Diptera), P. xylostella (Lepidoptera), Pediculus humanus (Phthiraptera), and Tribolium castaneum (Coleoptera), and 1 mite species (Tetranychus urticae) in the order Trombidiformes were downloaded from Ensembl. For genes with alternative splice variants, the longest transcripts were selected. We used Treefam [63] to define a gene family as a group of genes that descended from a single gene in the last common ancestor [64]. Phylogenetic trees were constructed from universal single-copy orthologues using maximum likelihood methods. The BRMC approach was used to estimate the species divergence time using the programme MULTIDIVTIME [65], which was implemented using the Thornian Time Traveller (T3) package (ftp://abacus.gene.ucl. ac.uk/pub/T3/).

\section{Gene family analysis}

Coding sequences and protein data of the above 18 insects and one mite (T. urticae) were used as database. For genes with alternative splice variants, the longest transcripts were selected. We used Treefam [63] to define a gene family as a group of genes that descended from a single gene in the last common ancestor [64]. We used CAFÊ [27] to identify gene family expansions and contractions in this study.

\section{Statistical analyses}

No statistical methods were used to predetermine sample sizes. Experiments were not randomized. The investigators were not blinded to allocation during experiments or outcome assessment. 


\section{Supplementary information}

Supplementary information accompanies this paper at https://doi.org/10. 1186/s12864-019-6266-0.

Additional file 1: Figure S1. Seventeen-k-mer estimation of $C$. vestalis and $D$. collaris genome size. (A) The genome size of $C$. vestalis was estimated to be $203 \mathrm{Mb}$ based on reads from $170 \mathrm{bp}$ and $500 \mathrm{bp}$ insertsize libraries. (B) The genome size of $D$. collaris was estimated to be 408 $\mathrm{Mb}$ based on reads from $170 \mathrm{bp}$ and $500 \mathrm{bp}$ insert size libraries. Figure S2. GC content and sequencing depth of $C$. vestalis (A) and D. collaris (B). $10 \mathrm{~Kb}$ non-overlapping sliding windows for $C$. vestalis, and $20 \mathrm{~Kb}$ nonoverlapping sliding windows for D. collaris were used to calculate the GC content and average depth among the windows. Figure S3. Comparison of amino acids biosynthesis between $C$. vestalis and $P$. xylostella. Three colors were used to show the difference between the two species on the pathway map of biosynthesis of amino acids (ko01230). Permission to use this pathway map image was kindly granted by the KEGG curators [61]. Table S1. Summary statistics of whole-genome sequencing data of $C$. vestalis and D. collaris. Table S2. Summary statistics of filtered data of C. vestalis and D. collaris. Table S3. Statistics of the genome assembly of $C$. vestalis and D. collaris. Table S4. Transcriptome sequence map to the genome assemblies of $C$. vestalis and D. collaris. Table S5. General statistics of predicted protein-coding genes for $C$. vestalis. Table S6. General statistics of predicted protein-coding genes for $D$. collaris. Table S7. Statistics of function annotation for $C$. vestalis and D. collaris. Table S8. TE statistics for $C$. vestalis, D. collaris and other insect genomes. Table S10. Microsynteny of $C$. vestalis and D. collaris genomes. Table S11. Statistics of syntonic regions of $C$. vestalis and D. collaris genomes compare to other insects. Table S13. Comparisons of the immunerelated genes among several arthropod species. Table S15. Comparisons of the detoxification genes among several arthropod species.

Additional file 2: Table S9. miRNAs in C. vestalis and D. collaris.

Additional file 3: Table S12. Expanded and reduced gene families in C. vestalis and $D$. collaris relative to other arthropods.

Additional file 4: Table S14. Expression level of immune and detoxification genes in C. vestalis and D. collaris.1. Kanehisa M, Goto S: KEGG: kyoto encyclopedia of genes and genomes. Nucleic Acids Res. 2000; 28(1): 27-30.

\section{Abbreviations}

BRMC: Bayesian relaxed molecular clock; BUSCO: Benchmarking Universal Single-Copy Orthologs; CvBV: Cotesia vestalis bracovirus; NEPs: Neprilysins; PDVs: Polydnaviruses; PO: Phenoloxidase; SMRT: Single Molecule Real-Time sequencing; TEs: Transposable elements; TRF: Tandem Repeats Finder

\section{Acknowledgments}

We thank Drs. Dicke Marcel, JH Werren and Shu-Sheng Liu for discussions and suggestions during the study.

\section{Authors' contribution}

Consortium leader: XXC.; Manuscript design: XXC, JHH., MS, XQY, ZZW, FL, MRS; Manuscript writing: XXC, FL, RS, ZZW, MS, XQY, FP.; P. xylostella, C. vestalis, and D. collaris population maintenance: JNZ, JYZ, LQZ, Y.W, YNZ, JY; Collection of DNA/RNA samples for genome, transcriptome: XQY, LQZ, ZHW, QJG, YY, SJW, RMH; Sequencing, assembling and annotation of genome and transcriptome: HQX, XXH, CLY, DHG; Table, figure and supplementary information: ZZW, XQY, MS, WCJ, XXH. All authors reviewed and approved the final version of the manuscript.

\section{Funding}

This work was supported by funds from the Chinese National Key Project for Basic Research (973 Project) (grant 2013CB127600), the Key Program of National Natural Science Foundation of China (grant 31230068, 31630060), the National Natural Science Foundation (31501700), and the National Science Fund for Innovative Research Groups (31321063). The funding bodies played no role in the design of the study and collection, analysis, and interpretation of data, nor in writing the manuscript.

\section{Availability of data and materials}

The filtered data used for de novo assembly were deposited into NCBI under SRA: SRR6356304, SRR6356303, SRR6356306, SRR6356305, SRR6356302, SRR6356301 (Illumina HiSeq 2000) and SRR6513317 (PacBio Sequel), associated with BioProject PRJNA307296 and BioSample SAMN04378091 for C. vestalis; SRR6346143, SRR6346142, SRR6346141, SRR6346140, SRR6346146, SRR6346145, SRR6346144 (Illumina HiSeq 2000) and SRR6513331 (PacBio Sequel), associated with BioProject PRJNA307299 and BioSample SAMN04378100 for D. collaris. The genome assembly data have also been deposited at DDBJ/EMBL/GenBank under accession numbers LQNH00000000 (C. vestalis) and LQNJ00000000 (D. collaris). The version described in this paper is the first version. The annotated data are available at the Waspbase website: http://www.insect-genome.com/waspbase/download/download_ content.php?downloads_type=Genome. The transcriptomic data are also available at the Waspbase website with a different directory: http://www. insect-genome.com/waspbase/download/download_content. php?downloads_type=Transcript.

Ethics approval and consent to participate Not applicable.

\section{Consent for publication}

Not applicable.

\section{Competing interests}

The authors declare that they have no competing interests.

\section{Author details}

${ }^{1}$ Institute of Insect Sciences, College of Agriculture and Biotechnology, Zhejiang University, Hangzhou 310058, China. ${ }^{2}$ Ministry of Agriculture Key Lab of Molecular Biology of Crop Pathogens and Insect Pests, Zhejiang University, Hangzhou 310058, China. ${ }^{3}$ State Key Lab of Rice Biology, Zhejiang University, Hangzhou 310058, China. ${ }^{4}$ BGI-Tech, BGl-Shenzhen, Shenzhen 518083, China. ${ }^{5}$ Xingzhi College, Zhejiang Normal University, Jinhua 321000, Zhejiang, China. ${ }^{6}$ School of Physical Science and Technology, Shanghai Tech University, Shanghai 201210, China. ${ }^{7}$ Institute of Plant and Environmental Protection, Beijing Academy of Agriculture and Forestry Sciences, Beijing 100097, China. ${ }^{8}$ Dipartimento di Agraria, Laboratorio di Entomologia "E. Tremblay", Università di Napoli "Federico II", Via Università 100, 80055 Portici, NA, Italy. 'Department of Entomology, University of Georgia, Athens, GA 30602, USA.

Received: 23 November 2018 Accepted: 5 November 2019

Published online: 21 November 2019

\section{References}

1. Vinson SB. Host selection by insect parasitoids. Annu Rev Entomol. 1976;21: 109-33.

2. Strand MR, Pech LL. Immunological basis for compatibility in parasitoid host relationships. Annu Rev Entomol. 1995:40:31-56.

3. Kraaijeveld AR, Godfray HCJ. Trade-off between parasitoid resistance and larval competitive ability in Drosophila melanogaster. Nature. 1997;389(6648): 278-80

4. Whitfield JB. Phylogeny and evolution of host-parasitoid interactions in hymenoptera. Annu Rev Entomol. 1998;43:129-51.

5. Tylianakis JM, Tscharntke T, Lewis OT. Habitat modification alters the structure of tropical host-parasitoid food webs. Nature. 2007; 445(7124):202-5

6. DeBach P. Biological control of insect pests and weeds. New York: Chapman and Hall; 1964.

7. Pennacchio F, Strand MR. Evolution of developmental strategies in parasitic hymenoptera. Annu Rev Entomol. 2006;51:233-58.

8. Asgari S, Rivers DB. Venom proteins from endoparasitoid wasps and their role in host-parasite interactions. Annu Rev Entomol. 2011;56:313-35.

9. Strand MR, Burke GR. Polydnavirus-wasp associations: evolution, genome organization, and function. Curr Opin Virol. 2013;3(5):587-94.

10. Herniou EA, Huguet E, Theze J, Bezier A, Periquet G, Drezen JM. When parasitic wasps hijacked viruses: genomic and functional evolution of polydnaviruses. Philos T R Soc B. 2013;368(1626):20130051.

11. Bezier A, Annaheim M, Herbiniere J, Wetterwald C, Gyapay G, BernardSamain S, Wincker P, Roditi I, Heller M, Belghazi M, et al. Polydnaviruses of 
braconid wasps derive from an ancestral nudivirus. Science. 2009;323(5916): 926-30.

12. White JA, Giorgini M, Strand MR, Pennacchio F. Arthropod endosymbiosis and evolution. Berlin Heidelberg: Springer; 2013.

13. Strand MR. Teratocytes and their functions in parasitoids. Curr Opin Insect Sci. 2014;6:68-73.

14. Werren JH, Richards S, Desjardins CA, Niehuis O, Gadau J, Colbourne JK, Beukeboom LW, Desplan C, Elsik CG, Grimmelikhuijzen CJ, et al. Functional and evolutionary insights from the genomes of three parasitoid Nasonia species. Science. 2010;327(5963):343-8.

15. Burke GR, Walden KKO, Whitfield JB, Robertson HM, Strand MR. Whole genome sequence of the parasitoid wasp Microplitis demolitor that harbors an endogenous virus mutualist. G3-Genes Genom Genet. 2018; 8(9):2875-80.

16. Geib SM, Liang GH, Murphy TD, Sim SB. Whole genome sequencing of the braconid parasitoid wasp Fopius arisanus, an important biocontrol agent of pest tepritid fruit flies. G3-Genes Genom Genet. 2017;7(8):2407-11.

17. Furlong MJ, Wright DJ, Dosdall LM. Diamondback moth ecology and management: problems, progress, and prospects. Annu Rev Entomol. 2013; 58:517-41.

18. You MS, Yue Z, He WY, Yang XH, Yang G, Xie M, Zhan DL, Baxter SW, Vasseur L, Gurr GM, et al. A heterozygous moth genome provides insights into herbivory and detoxification. Nat Genet. 2013;45(2):220-5.

19. Bai SF, Cai DZ, Li X, Chen XX. Parasitic castration of Plutella xylostella larvae induced by polydnaviruses and venom of Cotesia vestalis and Diadegma semiclausum. Arch Insect Biochem Physiol. 2009;70(1):30-43.

20. Ibrahim AMA, Kim Y. Parasitism by Cotesia plutellae alters the hemocyte population and immunological function of the diamondback moth, Plutella xylostella. J Insect Physiol. 2006;52(9):943-50.

21. Zhao W, Shi M, Ye XQ, Li F, Wang XW, Chen XX. Comparative transcriptome analysis of venom glands from Cotesia vestalis and Diadromus collaris, two endoparasitoids of the host Plutella xylostella. Sci Rep. 2017;7:1298.

22. Eid J, Fehr A, Gray J, Luong K, Lyle J, Otto G, Peluso P, Rank D, Baybayan P, Bettman B. Real-time DNA sequencing from single polymerase molecules. Science. 2009;323(5910):133-8.

23. Richards S. Arthropod Genome Sequencing and Assembly Strategies. In: Brown SJ, Pfrender ME, editors.Insect Genomics: Methods and Protocols. New York: Springer New York; 2019. p. 1-14.

24. Luo RB, Liu BH, Xie YL, Li ZY, Huang WH, Yuan JY, He GZ, Chen YX, Pan Q, Liu YJ, et al. SOAPdenovo2: an empirically improved memory-efficient shortread de novo assembler. Gigascience. 2012;1:18.

25. Simao FA, Waterhouse RM, loannidis P, Kriventseva EV, Zdobnov EM BUSCO: assessing genome assembly and annotation completeness with single-copy orthologs. Bioinformatics. 2015;31(19):3210-2.

26. Zhang HC, Rasnitsyn AP. Some ichneumonids (Insecta, Hymenoptera, Ichneumonoidea) from the upper Mesozoic of China and Mongolia. Cretac Res. 2003;24(2):193-202.

27. De Bie T, Cristianini N, Demuth JP, Hahn MW. CAFE: a computational tool for the study of gene family evolution. Bioinformatics. 2006;22(10):1269-71.

28. Yin C, Shen G, Guo D, Wang S, Ma X, Xiao H, Liu J, Zhang Z, Liu Y, Zhang Y, et al. InsectBase: a resource for insect genomes and transcriptomes. Nucleic Acids Res. 2016:44(D1):D801-7.

29. Jackson AP, Otto TD, Aslett M, Armstrong SD, Bringaud F, Schlacht A, Hartley C, Sanders M, Wastling JM, Dacks JB, et al. Kinetoplastid phylogenomics reveals the evolutionary innovations associated with the origins of parasitism. Curr Biol. 2016;26(2):161-72.

30. Bland ND, Pinney JW, Thomas JE, Turner AJ, Isaac RE. Bioinformatic analysis of the neprilysin (M13) family of peptidases reveals complex evolutionary and functional relationships. BMC Evol Biol. 2008;8:16.

31. El-Amouri SS, Zhu H, Yu J, Marr R, Verma IM, Kindy MS. Neprilysin: an enzyme candidate to slow the progression of Alzheimer's disease. Am J Pathol. 2008;172(5):1342-54.

32. Leissring MA, Farris W, Chang AY, Walsh DM, Wu XN, Sun XY, Frosch MP, Selkoe DJ. Enhanced proteolysis of beta-amyloid in APP transgenic mice prevents plaque formation, secondary pathology, and premature death. Neuron. 2003;40(6):1087-93.

33. Burrell M, Henderson SJ, Ravnefjord A, Schweikart F, Fowler SB, Witt S, Hansson KM, Webster Cl. Neprilysin inhibits coagulation through proteolytic inactivation of fibrinogen. PLoS One. 2016;11(7):e0158114.

34. Kanost MR, Jiang HB. Clip-domain serine proteases as immune factors in insect hemolymph. Curr Opin Insect Sci. 2015;11:47-55.
35. Pichon A, Bezier A, Urbach S, Aury JM, Jouan V, Ravallec M, Guy J, Cousserans F, Theze J, Gauthier J, et al. Recurrent DNA virus domestication leading to different parasite virulence strategies. Sci Adv. 2015;1(10): e1501150.

36. Asgari S, Reineke A, Beck M, Schmidt O. Isolation and characterization of a neprilysin-like protein from Venturia canescens virus-like particles. Insect Mol Biol. 2002;11(5):477-85

37. Chiorazzi M, Rui LX, Yang YD, Ceribelli M, Tishbi N, Maurer CW, Ranuncolo SM, Zhao H, Xu WH, Chan WCC, et al. Related F-box proteins control cell death in Caenorhabditis elegans and human lymphoma. P Natl Acad Sci USA. 2013;110(10):3943-8.

38. Yuan ZQ, Becker EBE, Merlo P, Yamada T, DiBacco S, Konishi Y, Schaefer EM, Bonni A. Activation of FOXO1 by Cdk1 in cycling cells and postmitotic neurons. Science. 2008;319(5870):1665-8.

39. Zhao L, Wang $P$, Hou H, Zhang H, Wang Y, Yan S, Huang Y, Li H, Tan J, Hu A, et al. Transcriptional regulation of cell cycle genes in response to abiotic stresses correlates with dynamic changes in histone modifications in maize. PLoS One. 2014;9(8):e106070.

40. Pennacchio F, Caccia S, Digilio MC. Host regulation and nutritional exploitation by parasitic wasps. Curr Opin Insect Sci. 2014;6:74-9.

41. Barribeau SM, Sadd BM, du Plessis L, Brown MJF, Buechel SD, Cappelle K, Carolan JC, Christiaens O, Colgan TJ, Erler S, et al. A depauperate immune repertoire precedes evolution of sociality in bees. Genome Biol. 2015;16:83.

42. Gao F, Gu QJ, Pan J, Wang ZH, Yin CL, Li F, Song QS, Strand MR, Chen XX, Shi M. Cotesia vestalis teratocytes express a diversity of genes and exhibit novel immune functions in parasitism. Sci Rep. 2016;6:26967.

43. Baehrecke EH. How death shapes life during development. Nat Rev Mol Cell Biol. 2002;3(10):779-87.

44. Lin Z, Cheng Y, Wang RJ, Du J, Volovych O, Li JC, Hu Y, Lu ZY, Lu ZQ, Zou Z. A metalloprotease homolog venom protein from a parasitoid wasp suppresses the toll pathway in host hemocytes. Front Immunol. 2018;9:2301.

45. Berenbaum MR, Johnson RM. Xenobiotic detoxification pathways in honey bees. Curr Opin Insect Sci. 2015;10:51-8.

46. Li XC, Schuler MA, Berenbaum MR. Molecular mechanisms of metabolic resistance to synthetic and natural xenobiotics. Annu Rev Entomol. 2007;52:231-53

47. Ode PJ. Plant chemistry and natural enemy fitness: effects on herbivore and natural enemy interactions. Annu Rev Entomol. 2006;51:163-85.

48. Wang ZZ, Ye XQ, Shi M, Li F, Wang ZH, Zhou YN, Gu QJ, Wu XT, Yin CL, Guo $\mathrm{DH}$, et al. Parasitic insect-derived miRNAs modulate host development. Nat Commun. 2018;9(1):2205

49. Lagos-Quintana M, Rauhut R, Lendeckel W, Tuschl T. Identification of novel genes coding for small expressed RNAs. Science. 2001;294(5543):853-8.

50. Friedlander MR, Mackowiak SD, Li N, Chen W. Rajewsky N: miRDeep2 accurately identifies known and hundreds of novel microRNA genes in seven animal clades. Nucleic Acids Res. 2012:40(1):37-52.

51. Koren S, Walenz BP, Berlin K, Miller JR, Bergman NH, Phillippy AM. Canu: scalable and accurate long-read assembly via adaptive k-mer weighting and repeat separation. Genome Res. 2017;27(5):722-36.

52. Benson G. Tandem repeats finder: a program to analyze DNA sequences. Nucleic Acids Res. 1999;27(2):573-80.

53. Tempel S. Using and understanding RepeatMasker. Methods Mol Biol. 2012; 859:29-51.

54. Altschul SF, Gish W, Miller W, Myers EW, Lipman DJ. Basic local alignment search tool. J Mol Biol. 1990;215(3):403-10.

55. Birney E, Clamp M, Durbin R. GeneWise and genomewise. Genome Res. 2004;14(5):988-95.

56. Ghosh S, Chan CK. Analysis of RNA-Seq data using topHat and cufflinks. Methods Mol Biol. 2016;1374:339-61.

57. Stanke M, Waack S. Gene prediction with a hidden Markov model and a new intron submodel. Bioinformatics. 2003;19:li215-25.

58. Salamov AA, Solovyev W. Ab initio gene finding in Drosophila genomic DNA. Genome Res. 2000;10(4):516-22

59. Bairoch A, Apweiler R. The SWISS-PROT protein sequence database and its supplement TrEMBL in 2000. Nucleic Acids Res. 2000;28(1):45-8.

60. Mulder N, Apweiler R. InterPro and InterProScan: tools for protein sequence classification and comparison. Methods Mol Biol. 2007;396:59-70.

61. Ashburner M, Ball CA, Blake JA, Botstein D, Butler H, Cherry JM, Davis AP, Dolinski K, Dwight SS, Eppig JT, et al. Gene ontology: tool for the unification of biology. Nat Genet. 2000;25(1):25-9. 
62. Kanehisa M, Goto S. KEGG: Kyoto encyclopedia of genes and genomes. Nucleic Acids Res. 2000;28(1):27-30

63. Li H, Coghlan A, Ruan J, Coin LJ, Heriche JK, Osmotherly L, Li R, Liu T, Zhang $Z$, Bolund $L$, et al. TreeFam: a curated database of phylogenetic trees of animal gene families. Nucleic Acids Res. 2006;34(Database issue):D572-80.

64. Li R, Fan W, Tian G, Zhu H, He L, Cai J, Huang Q, Cai Q, Li B, Bai Y, et al. The sequence and de novo assembly of the giant panda genome. Nature. 2010; 463(7279):311-7.

65. Wikstrom N, Savolainen V, Chase MW. Evolution of the angiosperms: calibrating the family tree. Proc Biol Sci. 2001;268(1482):2211-20.

\section{Publisher's Note}

Springer Nature remains neutral with regard to jurisdictional claims in published maps and institutional affiliations.

- fast, convenient online submission

- thorough peer review by experienced researchers in your field

- rapid publication on acceptance

- support for research data, including large and complex data types

- gold Open Access which fosters wider collaboration and increased citations

- maximum visibility for your research: over $100 \mathrm{M}$ website views per year

At BMC, research is always in progress.

Learn more biomedcentral.com/submissions 